Horizons philosophiques

\title{
Commentaire : " une affaire de dignité ou la bioéthique n’est pas un roman "
}

\section{Marie-Hélène Parizeau}

Volume 4, numéro 2, printemps 1994

Médecines impossibles?

URI : https://id.erudit.org/iderudit/800947ar

DOI : https://doi.org/10.7202/800947ar

Aller au sommaire du numéro

Éditeur(s)

Collège Édouard-Montpetit

ISSN

1181-9227 (imprimé)

1920-2954 (numérique)

Découvrir la revue

Citer ce document

Parizeau, M.-H. (1994). Commentaire : « une affaire de dignité ou la bioéthique n'est pas un roman ». Horizons philosophiques, 4(2), 35-42.

https://doi.org/10.7202/800947ar d'utilisation que vous pouvez consulter en ligne.

https://apropos.erudit.org/fr/usagers/politique-dutilisation/ 


\section{COMMENTAIRE : «UNE AFFAIRE DE DIGNITÉ OU LA BIOÉTHIQUE N'EST PAS UN ROMAN»}

Une histoire d'escalade technique, de relation affective forte entre soignants et malades, de rapports difficiles entre professionnels, d'envolées existentielles, de pistes de réflexion éthique, telle est la trame de ce récit de vies. Une histoire qui nous place au cœur de l'action, de l'émotion, d'une rationalité opératoire implacable, illustrée par une quantité d'individus attachant par leur souffrance ou par leur volonté de bien faire. Nous sommes vite entraînés à la suite de ces personnages, par des actes qui s'enchaînent, liant les destins par la mort et la vie, et nous nous posons nécessairement des questions d'ordre éthique, sur le sens, les moyens et les finalités de ce qui est fait, dit ou pensé.

Ces questions sont au cœur de la bioéthique et nous mènent au seuil de la réflexion en philosophie morale. Car, dans cette histoire, tout reste à être réfléchi, clarifié, analysé et élaboré au plan théorique. Ce récit se situe dans le registre littéraire de la nouvelle et non dans l'histoire de cas classique de bioéthique, en particulier de l'éthique clinique. Mon propos est de montrer les limites du récit dans le contexte de la bioéthique, et de proposer une analyse des principaux dilemmes éthiques contenus dans l'histoire afin d'illustrer l'importance d'une démarche rigoureuse et systématique dans la prise de décision éthique pour éviter le subjectivisme émotif.

Le mérite particulier du récit est de mettre le lecteur en situation : il devient vite pris par l'histoire, les personnages, les sentiments, comme si lui-même participait à l'action. Le lecteur devient tour à tour la jeune Inuk Martha Tulugak qui meurt, le $\mathrm{Dr}$ Joseph Stein qui veut à tout prix prélever les reins pour sauver une autre jeune femme, Sylvie Marois, ou encore le professeur 
Marc Granger avec ses interrogations sans fin. Le récit permet de pénétrer cette épaisseur de vies par le biais de l'évocation des sentiments avec le support d'une trame linéaire (un début et une fin). Avec le récit, le lecteur acquiert une familiarité d'un certain type de situation qui, au départ, peut lui être parfaitement étranger. Le récit a cette force évocatrice de la réalité donc une puissance de conversion qui, dans l'histoire Une affaire de dignité, appelle l'éthique.

Utilisant le vocabulaire de Paul Ricœur, je dirais que cet appel à l'éthique est la référence dans le récit, "à savoir le pouvoir de la métaphore de projeter et de révéler un monde ${ }^{1}$ ». En d'autres termes, l'histoire qui nous est proposée ouvre le lecteur à l'éthique. Mais il n'offre ni une réflexion, ni un raisonnement éthique systématique. L'œuvre littéraire, qu'elle soit sous forme de poème, d'essai ou de fiction en prose, utilise le langage métaphorique, c'est-à-dire essentiellementpolysémique où les connotations sont libérées ${ }^{2}$. Le lecteur a donc le loisir et la possibilité de choisir selon son bon vouloir dans les significations implicites.

Ce récit ouvre donc sur l'éthique et sur toutes sortes de questions et réflexions morales plus ou moins étendues selon le lecteur. Quiconque est un peu familier des questions de bioéthique peut jouer ici le rôle du critique littéraire ${ }^{3}$ et s'attacher

1. Paul Ricoeur. La Métaphore vive, Paris, Seuil, 1975, p. 120.

2. "La signification explicite d'un mot est sa désignation; sa signification implicite, sa connotation. Dans le langage ordinaire, la "gamme complète des connotations" n'est jamais effectuée dans un contexte particulier; seule l'est une partie choisie de cette gamme : c'est la "connotation contextuelle" du mot. Dans certains contextes, les autres mots éliminent les connotations non désirables d'un mot donné; c'est le cas du langage technique et scientifique où tout est explicite. "Dans d'autres contextes, les connotations sont libérées : ce sont principalement ceux où le langage devient figuré et plus particulièrement métaphorique" ". (Ricoeur, p. 118)

3. Le rôle du critique littéraire est ici entendu dans le sens de celui de Richard Rorty, tel qu'il le décrit dans Contingency, Irony and Solidarity. "Le mot "littérature" recouvre désormais autant de genres de livres susceptibles d'avoir une pertinence morale, susceptibles de modifier le sentiment que nous avons de ce qui est important. L'application de cette expression n'a rien à voir avec la présence dans un livre de "qualités littéraires". Plutôt que de détecter et d'exposer de telles qualités, on attend désormais du critique qu'il facilite la réflexion morale en suggérant des révisions dans le canon des archétypes et conseillers moraux, mais aussi des manières d'apaiser les tensions au sein de ce canon ou, au besoin, de 
au sens du récit en dégageant les moments éthiques, en clarifiant les enjeux moraux et en proposant une réflexion éthique, c'est-à-dire sur la référence même du récit. Je me propose d'essayer rapidement ${ }^{4}$ de faire cet exercice et d'en montrer les limites qui sont intrinsèques à la forme même du récit.

Dans le récit dont le personnage principal est la jeune Inuk, Martha Tulugak ${ }^{5}$, on peut identifier trois dilemmes éthiques (des conflits de valeurs) principaux qui correspondent à autant de moments où une décision détermine la suite de l'action.

Le premier dilemme éthique se situe au tout début du récit, lorsque Richard Munro fait le diagnostic de l'anévrysme qui s'accompagne d'un mauvais pronostic et décide d'intervenir ( «tentative des vivants pour permettreà une comateuse d'échapper à la mort") par la technique en usage (Décadron) et par un transfert (accessibilité aux soins). La justification morale de Munro qui semble se dégager plus loin serait vraisemblablement de "charger sa vie de significations". Poussons un peu plus loin l'analyse. La médecine se situe dans le registre de l'agir pour le bien du malade (principe de bienfaisance ${ }^{6}$ ). La médecine se détermine intrinsèquement par un a priori en faveur de la vie - de plus, dans la sensibilité contemporaine,

les accentuer" (Richard Rorty, Contingence, ironie et solidarité, tr. française P-E Dauzat, Paris, Armand Colin, 1993, p. 120). Sans adhérer à la conception de la philosophie de Rorty qui la considère comme narration plutôt que système, en fait essentiellement poétique, je me retrouve néanmoins, dans le présent exercice, dans la situation du critique littéraire tel que le décrit Rorty.

4. Compte tenu des limites imposées dans le cadre de cet article, je focaliserai sur les problèmes éthiques qui m'apparaissent importants en bioéthique.

5. Le personnage de Martha Tulugak est d'ailleurs étonnant d'imprécision; on ne sait même pas à quoi elle ressemble physiquement, bref on a de la difficulté à se la "représenter". Dans une analyse de cas en bioéthique, l'histoire bio-bliographique d'une personne est essentielle parmi les critères facilitant la prise de décision. Elle permet de mieux situer la personne dans son environnement naturel (est-elle seule ou a-t-elle un conjoint, des enfants, des parents? Quel est son rang dans la fratrie? A-t-elle une occupation principale ou un travail? Quel genre de vie mène-t-elle? Estelle croyante et de quelle confession? A-t-elle une histoire médicale antérieure? etc.)

6. Le principe de bienfaisance se définit de diverses façons qui témoignent d'une gradation de l'obligation morale : 1) le devoir de ne pas nuire; 2) le devoir de prévenir 
on supporte peu la mort d'un être jeune ${ }^{7}$. L'impératif technicien, quant à lui, énonce qu'il faut faire ce qui est techniquement possible et le risque est que le moyen prenne le pas sur la fin. Dans ce premier dilemme, Munro a décidé d'agir en fonction du bien de la malade (la vie), malgré l'incertitude médicale et parce qu'il dispose des moyens techniques de son action. II ne nous est pas possible ici, à partir des bribes d'informations médicales dont nous disposons dans ce récit, de savoir si Munro a pris, au plan strictement scientifique, la bonne décision. Prenons pour acquis qu'il a jugé de la situation clinique selon les canons des bonnes pratiques médicales en cours. Notons qu'il aurait pu prendre la décision de ne pas intervenir davantage sur la base de critères médicaux ou encore d'une expérience professionnelle plus étendue ${ }^{8}$.

Le récit est donc engagé par la décision de Munro dans le sens de l'intervention (deuxième dose de Décadron avec l'espoir au moins d'une stabilisation de l'état de la malade; chassécroisé avec l'avion-ambulance qui vient de Québec). Surgit ici le deuxième dilemme éthique. Nous voici à Kuujjuaq et la tension artérielle de la patiente monte anormalement. Pourtant, elle est embarquée dans l'avion, mais Martha Tulugak meurt au début du vol. Ici se décide toute la suite de l'histoire. Une décision technique importante va être prise : brancher le tube endotrachéal pour maintenir les fonctions vitales pour un certain temps. Le temps nécessaire au prélèvement d'un certain nombre d'organes car la façon dont est morte Martha, la

le mal ou la souffrance; 3) le devoir de supprimer le mal ou la souffrance; 4) le devoir de faire le bien d'autrui ou de promouvoir son bien. Globalement le principe de bienfaisance consiste à faire le bien d'autrui de façon plus ou moins directe. Voir la description du principe de bienfaisance p. 89 (entrée "consentement") dans LeS Mots de la bioéthique. Un vocabulaire encyclopédique, G. Hottois et M.-H. Parizeau (sous la direction), 1993, Bruxelles-Montréal, De Boeck-ERPI.

7. On peut se demander si la décision du médecin aurait été la même si, dans une situation analogue, la patiente avait été une personne âgée.

8. II est connu en médecine, que les jeunes médecins ont tendance à avoir des comportements plus interventionnistes au plan technique que les médecins ayant une expérience professionnelle plus longue. 
désigne tout naturellementau don d'organes. C'est en effet une candidate idéale au prélèvement d'organes, compte tenu de la cause de sa mort, de sa jeunesse et de son état de santé antérieur. On peut se demander si Nicole Gagné, l'infirmière et Paul Drouin, le médecin, ont réfléchi sur les suites possibles de cet acte ou s'ils ont tout simplement appliqué ce qu'on leur a appris, les techniques et les actes à poser dans ce type de situation, quelles que soient les circonstances. Ici, semble-t-il, c'est davantage le réflexe technique qui a prévalu. Au lieu d'avoir l'idée saugrenue de larguer le corps de Martha dans la toundra, pourquoi ne pas avoir rebroussé chemin et rendu le corps aux siens, en particulier à celui qui avait accompagné Martha dès le début, son frère, George?

Le jugement médical doit aussi être contextualisé. En d'autres termes, une décision médicale est aussi une décision éthique. Celle-ci fait intervenir à des degrés variables les circonstances (le contexte global). Je dois m'écarter de la critique littéraire et entrer directement sur le terrain de la philosophie morale pour faire un commentaire sur cette dernière affirmation. Il est difficile de tenir une position strictement déontologique en bioéthique, surtout dans les cas particuliers : on est tenu également de considérer aussi les principes "conséquentialistes ${ }^{9}$ ".

À ce moment du récit, tout lecteur attentif aura lui-même retracé toute une série de circonstances qui caractérisent très précisément le contexte de la prise de décision (le climat et

9. "Les deux autres principes pratiques - les principes conséquentialiste et déontologique - sont universalistes et représentent des obligations catégoriques. Le principe conséquentialiste exige que l'on fasse ce qui produira globalement le plus grand bien (la plus grande somme algébrique de bien et de mal) eu égard à tous ceux qui sont affectés par notre action ('utilitarisme est la version la plus connue de ce principe, version qui considère le bien comme la satisfaction du désir). Le principe déontologique exige que l'on ne fasse jamais certaines choses (ne pas respecter une promesse, dire des mensonges, tuer un innocent) à autrui, même s'il doit en résulter globalement un moindre bien ou un mal plus grand". (Charles Larmore, Modernité et Morale, Paris, PUF, 1993, p. 97). Charles Larmore plaide pour l'hétérogénéité de la morale et ouvre ainsi des pistes de réflexion intéressantes pour la bioéthique. 
l'éloignement, la civilisation inuit avec sa présence continue près de la malade, la religiosité, les derniers sacrements, etc.). Àl'univers technicien, s'oppose un monde symbolique, chacun ayant sa propre logique et ses propres valeurs. L'histoire bascule dans la logique technicienne à partir du deuxième dilemme.

Le troisième dilemme s'ouvre sur l'univers des miracles de la médecine contemporaine, dans lequel, grâce à des moyens techniques sophistiqués, des équipes médicales sauvent des vies humaines d'une mort certaine. On notera le vocabulaire quasi-religieux qu'utilise notre narrateur pour décrire le contexte - le songe du chirurgien, l'éblouissement, le sourire de Sylvie Marois que l'on pourrait surnommer l'ange - - Le troisième dilemme contrairement au second qui peut passer inaperçu, se présente sous la forme d'un conflit ouvert entre le chirurgien qui veut sauver la vie de Sylvie et le directeur des soins professionnels de l'hôpital qui représente ici l'autorité sociale et la loi. Le chirurgien va-t-il transgresser la loi qui exige le consentement de la famille pour un prélèvement d'organes? Le chirurgien va-t-il respecter la limite qu'a posée la société en matière de prélèvement d'organes et qui touche aux représentations du respect du corps et au principe de l'autonomie de la volonté de la personne? Ou va-t-il juger qu'au nom du principe de bienfaisance (faire le bien de Sylvie dont la vie pourrait être ainsi sauvée), il doit passer outre à la loi? Ultimement, une décision éthique est une décision individuelle : chacun est face à sa conscience morale, aux raisons de son choix moral, engageant ainsi sa propre responsabilité morale. Cette responsabilité en est une vis-à-vis de soi-même mais aussi vis-à-vis de la société. Notons que, dans l'histoire de la médecine, certains médecins n'ont pas craint de s'opposer à la loi, jusqu'à faire de la prison au nom de certaines valeurs ${ }^{10}$.

10. L'histoire de l'avortement est assez claire de l'engagement moral de certains médecins pour le libre accès des femmes à l'avortement. L'euthanasie aujourd'hui connaîtégalementl'engagement de certains médecins en faveur d'un encadrement juridique favorable à l'euthanasie. 
L'identification des trois dilemmes éthiques de ce récit permet d'illustrer trois éléments importants de la prise de décision médicale dans le contexte d'un conflit moral : 1) l'évaluation du jugement clinique à partir de critères strictement scientifiques; 2) les conséquences d'un acte médical en fonction de l'appréciation contextuelle de la situation; 3) le rapport de la décision médicale aux normes sociales et juridiques. Dans les analyses de cas clinique classique de bioéthique, ces trois éléments sont abordés systématiquement, de même que sont clarifiés les principes et les valeurs en jeu dans tout dilemme éthique. Il est supposé, dans cette approche, un cognitivisme et une rationalité dans la prise de décision éthique. En d'autres termes, une décision médicale, dont découlent des conséquences pratiques et qui implique un choix de valeurs explicite, devrait être justifiée par des arguments d'ordre rationnel dans un espace de communication. Ce modèle argumentatif et communicationnel est directement emprunté à Jürgen Habermas ${ }^{11}$. Tout choix d'agir ou de ne pas agir doit comporter des raisons auxquelles les autres (le patient, l'équipe soignante, les proches, voire la société) pourraient adhérer de façon consensuelle.

On peut s'étonner que le professeur Marc Granger n'a pas adopté cette méthode d'analyse plus rigoureuse avec son interlocuteur si néophyte soit-il. Aurait-il lu Engelhardt plus attentivement qu'il aurait saisi la tentative néo-kantienne de fonder en raison une entente pacifique entre les communautés pluralistes en tentant de définir le sujet moral ${ }^{12}$. II aurait pu ouvrir ses livres de philosophie, revoir les arguments universalistes de Kant et approcher les débats contemporains sur les éthiques

11. On se rapportera en particulier à : 1- Habermas J., Morale et communication, tr. fr. C. Bouchindhomme, Paris, Cerf, 1986. Moralbewusstsein und Kommunikatives Handeln, Frankfurt, Suhrkamp, 1983.2- Habermas J., De l'éthique de la discussion, tr. fr. M. Hunyadi, Paris, Cerf, 1992. Erlauterungen zur Diskursethik, Frankfurt, Suhrkamp, 1991.

12. Même si on ne partage pas les arguments de T. Engelhardt sur le statut de la personne humaine, il faut saluer un des encore trop rares efforts théoriques pour penser les fondements de la bioéthique. 
procédurales et les éthiques de la vie bonne ${ }^{13}$. Une telle perspective aurait pu lui permettre d'aborder le débat dans des termes différents, de façon moins émotive et subjectiviste. Car, dans ses réflexions solitaires, il ne dépasse guère le niveau du sens moral commun et l'on s'attend à quelque chose de plus (au moins une clarification du problème éthique) d'un professeur ayant étudié le champ de la bioéthique.

Si le récit a une valeur heuristique - et Hans Jonas ${ }^{14}$ a bien montré qu'il pouvait y avoir une utilité à convertir à l'éthique - il n'en reste pas moins que le récit a ses limites en ce qu'il ne donne pas les outils réflexifs et méthodologiques d'une aide à la prise de décision d'un dilemme éthique.

Chemin faisant, le critique littéraire est redevenu un philosophe critique!

\author{
Marie-Hélène Parizeau \\ Faculté de philosophie \\ Université Laval
}

13 Le lecteur pourra se reporter entre autre aux articles de Jean-Marc Ferry, "Sur la responsabilité à l'égard du passé. L'éthique de la discussion comme éthique de la rédemption", Hermes (1991) 10, p. 125-137 et d'Anne Fagot-Largeault, "La réflexion philosophique en bioéthique" dans Les Fondements de la bioéthique, M.- H. Parizeau ed., Bruxelles/Montréal, De Boeck-ERPI, 1992, p. 11-26, ainsi qu'au livre de Gilbert Hottois, Le Paradigme bioéthique, Bruxelles/Montréal, De Boeck-ERPI, 1990.

14. Jonas $\mathrm{H}$. The Imperative of Responsability. In Search of an Ethics for the Technological Age, Chicago, The Chicago University Press, 1984, tr. française Jean Greisch, Le Principe de responsabilité. Une éthique pourla civilisation technologique, Paris, Cerf, 1991. 